\title{
Rituximab Therapy Leads to Reduced Imprints of Receptor Revision in Immunoglobulin $\kappa$ and $\lambda$ Light Chains
}

\author{
ARUMUGAM PALANICHAMY, KHALID MUHAMMAD, PETRA ROLL, STEFAN KLEINERT, THOMAS DÖRNER, \\ and HANS-PETER TONY
}

\begin{abstract}
Objective. Transient B cell depletion by rituximab (RTX) has become a specific treatment of rheumatoid arthritis (RA). Although phenotypic repopulation kinetics of B cell subsets are well documented, precise molecular analyses of the reconstituting immunoglobulin (Ig) genes encoding the B cell receptor in RA are sparse.

Methods. A total of 708 individual CD19+CD27+ (memory) and CD19+CD27- (naive) B cells from 2 patients with RA were analyzed at baseline and 7 months after RTX at B cell repopulation. Ig light chain variable kappa $(\mathrm{V} \kappa)$ and lambda $(\mathrm{V} \lambda)$ light chain gene rearrangements were amplified, sequenced, and analyzed with a focus on receptor revision.

Results. The naive as well as the memory repertoire repopulated polyclonally with diverse use of variable light chain gene families and minigenes. During the reconstitution phase, B cells used sig-

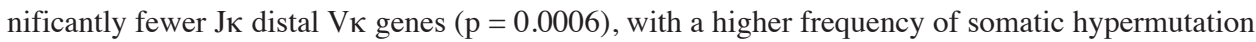
of rearrangements employing J 5 compared to baseline in memory B cells. The use of V $\lambda$ rearrangements in regenerating $\mathrm{B}$ cells was also biased toward use of $\mathrm{V} \lambda$ genes of the proximal cassette. In general, reemerging CD27+ Ig light chain genes were substantially more highly mutated than before RTX therapy ( $<<0.0001$, baseline vs during reconstitution).

Conclusion. Our data indicate that RTX therapy leads to generation of distinct $\mathrm{V} \kappa / \mathrm{J} \kappa$ and $\mathrm{V} \lambda / \mathrm{J} \lambda$ gene repertoires consistent with replenishment of antigen-experienced B cells by germinal centers. At baseline, the imprints of receptor revision appeared to be more striking, which indicates that receptor revision is active in patients with RA and can be reduced by RTX. (First Release April 15 2012; J Rheumatol 2012;39:1130-8; doi:10.3899/jrheum.111513)
\end{abstract}

Key Indexing Terms:

RHEUMATOID ARTHRITIS IMMUNOGLOBULIN LIGHT CHAINS RECEPTOR REVISION

Rheumatoid arthritis (RA) is an immune-mediated systemic inflammatory disorder manifested by aggressive synovitis that leads to progressive cartilage degradation and bone erosion, which causes pain, joint deformity, and functional dis-

From the Department of Rheumatology and Clinical Immunology, Medizinische Klinik und Poliklinik II, University of Würzburg, Würzburg; and CC12, Institute of Transfusion Medicine and Immune Haematology, Charite Universitätsmedizin Berlin and DRFZ Berlin, University of Berlin, Berlin, Germany.

Supported by Wilhelm Sanderstiftung 2006.037.2, University of Würzburg, Würzburg, and project 14, SFB 650, Charite University, Berlin .

A. Palanichamy, PhD; K. Muhammad, PhD; P. Roll, MD; S. Kleinert, $M D$, Department of Rheumatology and Clinical Immunology,

Medizinische Klinik und Poliklinik II, University of Würzburg; T. Dörner, $M D, C C 12$, Institute of Transfusion Medicine and Immune Haematology, Charite Universitätsmedizin Berlin and DRFZ Berlin, University of Berlin; H-P. Tony, MD, Department of Rheumatology and Clinical Immunology, Medizinische Klinik und Poliklinik II, University of Würzburg.

A. Palanichamy and K. Muhammad share first authorship of this article.

Address correspondence to Dr. H-P. Tony, Department of Rheumatology and Clinical Immunology, Medizinische Klinik und Poliklinik II,

Oberdürrbacherstr. 6, 97080 Würzburg, Germany.

E-mail:Tony_H@medizin.uni-wuerzburg.de

Accepted for publication February 23, 2012. ability ${ }^{1,2}$. Whereas different cell types play pathogenic roles in RA, B cells have drawn particular attention ${ }^{3}$. B cells do not merely secrete autoantibodies including rheumatoid factor (RF) and antibodies against citrullinated peptides, but are also involved in secretion of proinflammatory cytokines, activating autoreactive $\mathrm{T}$ cells, and presenting and processing autoantigens, leading to infiltration of end organs ${ }^{4}$. B cells are able to rearrange specific antigenic receptors by receptor-editing and lack of central and/or peripheral tolerance contributes to their abnormal activation and proliferation in autoimmune diseases ${ }^{5,6}$. The importance of B cells in the pathophysiology of RA has been emphasized by therapeutic approaches using the anti-CD20 monoclonal antibody rituximab (RTX). RTX results in prolonged alleviation of clinical symptoms and inflammation and reduced erosive joint destruction ${ }^{7}$. We and other investigators have reported regeneration kinetics from $B$ cell depletion studies indicating that peripheral B cells repopulate between 6 months and 10 months after depletion with a majority of newly emerging immature $\mathrm{B}$ cells ${ }^{8,9}$.

The $\mathrm{B}$ cell repertoire is shaped by a complex set of genes that are modulated through different rearrangements, somat- 
ic hypermutation, and receptor revision. All these processes are involved in producing unique and diverse immunoglobulin (Ig) receptors and are highly regulated during ontogeny, development, and response to antigen. Selection and activation of autoreactive B cells in healthy individuals is avoided by a tolerance mechanism and the vast majority of naive autoreactive B cells are deleted through apoptosis in their premature stages ${ }^{10,11}$.

B cell antigen receptor revision involves secondary Ig gene rearrangement. Ig-transgenic mouse model studies identified receptor revision events as a relatively rare process capable of rescuing a small fraction of autoreactive $\mathrm{B}$ cells ${ }^{12}$. The $\mathrm{IgV}$ repertoire is shaped by a variety of selective and molecular events. Previous studies indicated the existence of disrupted receptor revision in $\mathrm{RA}^{13}$. However, no data are available analyzing the light chain repertoire under RTX, which might follow different expression profiles and could be exposed to distinct selection pressures.

We analyzed the molecular aspects of B cell receptor after transient $\mathrm{B}$ cell depletion in patients with RA, with a focus on modulation of $\mathrm{B}$ cell immunoglobulin $\mathrm{V \kappa}$ and $\mathrm{V} \lambda$ light chain gene rearrangements in $\mathrm{CD} 27$ - naive and CD27+ memory B cells. In particular, we analyzed the mutational pattern and Ig gene repertoire before B cell depletion and during the $\mathrm{B}$ cell regeneration phase (7 months) after a single dose of RTX.

\section{MATERIALS AND METHODS}

Patients. Informed consent was obtained from the patients according to a protocol approved by the ethics committee of the University of Würzburg and according to the Declaration of Helsinki. Patient 1 (female, 47 years old) and Patient 2 (female, 35 years old) had active RA.

Both patients were RF-positive and were diagnosed according to the American College of Rheumatology revised criteria for RA ${ }^{14}$. They were nonresponders to standard disease-modifying antirheumatic drugs. Patients were receiving a stable dose of methotrexate before receiving 2 treatments of $1000 \mathrm{mg}$ RTX, 2 weeks apart. Disease activity was determined by measuring the 28-joint Disease Activity Score, erythrocyte sedimentation rate, and C-reactive protein levels. Further disease variables are shown in Table 1. Both patients showed a clinical response during the study. Patient 2 experienced increasing disease activity at the second timepoint of analysis.

Single cell sorting. For separation of peripheral mononuclear cells, a blood sample suspension was centrifuged on a Ficoll-paque gradient (GE Healthcare, Munich, Germany) with subsequent washing steps in phosphate buffered saline (PBS). A single B cell sorting technique was per- formed as described ${ }^{15,16}$. In brief, B cells were stained with fluorescence-labeled monoclonal antibodies against B cell surface markers: CD19-APC and CD27-PE. Cell incubation with antibodies was performed at $4^{\circ} \mathrm{C}$ for $15 \mathrm{~min}$ in PBS $/ 0.5 \%$ bovine serum albumin (BSA). Individual B cells were sorted into 96-well plates containing lysis buffer using a FACS DiVA process (Beckton Dickinson). Lysis buffer consisted of DTT (Roche Diagnostics, Mannheim, Germany), RNasin (Promega), Oligo dT(15) primer (Promega), BSA (Sigma-Aldrich), Triton 10\% (EMD), and molecular grade $\mathrm{H}_{2} \mathrm{O}$.

cDNA synthesis. A mixture containing nucleotides, reverse transcriptase, and RT-polymerase chain reaction buffer from the Titan One Tube RT-PCR System (Roche) was added into each well of a 96-well plate containing sorted B cells. cDNA was synthesized by incubation at $50^{\circ} \mathrm{C}$ for $1 \mathrm{~h}$.

Nested polymerase chain reaction (PCR). Vк (families 1-6) and $\mathrm{V} \lambda$ (families 1-10) were amplified by nested PCR using family-specific primers. Reaction mixture, primers, and cycling conditions were as described ${ }^{17,18}$. PCR products were separated by electrophoresis on a $1.5 \%$ agarose gel. The products were purified further with a MinElute Gel Extraction kit (Qiagen, Hilden, Germany).

Sequencing and analysis. Gel-extracted $\mathrm{V} \kappa$ and $\mathrm{V} \lambda$ family products were further amplified using a BigDye Terminator Cycle Sequencing Ready Reaction kit (Perkin-Elmer Applied Biosystems, Weiterstadt, Germany) and 5 ' internal primers of each family followed by sequencing in an automated genetic analyzer, the ABI Prism 310 (Applied Biosystems). Sequence analysis was performed using the online program JOINSOLVER $^{\circledR 19}$ (US National Institutes of Health, National Institute of Arthritis and Musculoskeletal and Skin Diseases) and productively rearranged sequences were matched to the closest germline configuration in the ImMunoGeneTics information system database. Out of frame and nonproductive sequences with a stop codon were excluded from the analysis.

Statistical analysis. Statistical analyses were performed using GraphPad Prism 3.03 (GraphPad Software, San Diego, CA, USA). Frequencies of Vк and $\mathrm{V} \lambda$ families as well as $\mathrm{J} \kappa$ and $\mathrm{J} \lambda$ gene usage were compared using 2 -tailed Fisher's exact test. Chi-square test was used to compare the mutational frequencies, RGYW/WRCY mutations, and R/S mutations, while CDR3 lengths were compared by nonparametric Mann-Whitney U test. $P$ values $<0.05$ were considered statistically significant.

\section{RESULTS}

Distribution of $V \kappa$ and $V \lambda$ family repertoire. In order to identify potential therapy-related molecular modifications in circulating B cells, we studied the Ig light chain gene usage of 2 RA patients in detail. CD19+CD27+ memory and CD19+CD27- naive B cells from both patients used light chain gene rearrangements in similar proportions in their productive repertoires, with no significant differences. Therefore, we grouped the Ig sequences from both patients

Table 1. Laboratory and clinical variables of the patients treated with rituximab. B cell subsets were analyzed by flow cytometry. Results are shown as percentage of CD19+ lymphocytes.

\begin{tabular}{|c|c|c|c|c|c|c|c|c|c|}
\hline \multicolumn{10}{|l|}{ Patient 1} \\
\hline Baseline & 9 & 10 & 4.84 & 0.56 & 12 & 15.5 & 14.0 & 2.5 & 67.5 \\
\hline \multicolumn{10}{|l|}{ Patient 2} \\
\hline Baseline & 15 & 11 & 5.80 & 8.71 & 33 & 12.0 & 16.5 & 2.7 & 63.0 \\
\hline After RTX & 12 & 7 & 5.51 & 5.42 & 38 & 1.0 & 13.0 & 4.7 & 76.0 \\
\hline
\end{tabular}

SJC: swollen joint count; TJC: tender joint count; DAS: Disease Activity Score; CRP: C-reactive protein; ESR: erythrocyte sedimentation rate.

Personal non-commercial use only. The Journal of Rheumatology Copyright (C) 2012. All rights reserved. 
and examined the changes in the light chain repertoire in terms of variable (V) and joining (J) gene usage, CDR3 length, and mutational imprints.

Before depletion, Vк1 family members were significantly overexpressed, followed by $\mathrm{V} \kappa 2$ and $\mathrm{V} \kappa 3$ family members. VК4 family genes were observed at lower frequencies, whereas no $\mathrm{V} \kappa 5$ or $\mathrm{V} \kappa 6$ family members were found. During repletion after RTX, a number of changes of $\mathrm{V}$ family gene usage were observed. The frequency of occurrence of VК1 family members decreased $(63.5 \%$ vs $48 \%$; p < 0.05 ), while occurrence of $\mathrm{V} \kappa 3$ genes increased $(13.5 \%$ vs $26.5 \%$; $<<0.05$; Figure 1A). The frequencies of $\mathrm{V} \kappa 2$ and $\mathrm{V \kappa} 4$ family members before and after RTX were not statistically significantly different.

$\mathrm{V} \lambda$ light chain analysis showed that $\mathrm{V} \lambda 1,2$, and 3 family members were predominantly employed by the expressed repertoire before and after depletion with $\mathrm{V} \lambda 2$ genes, fol-
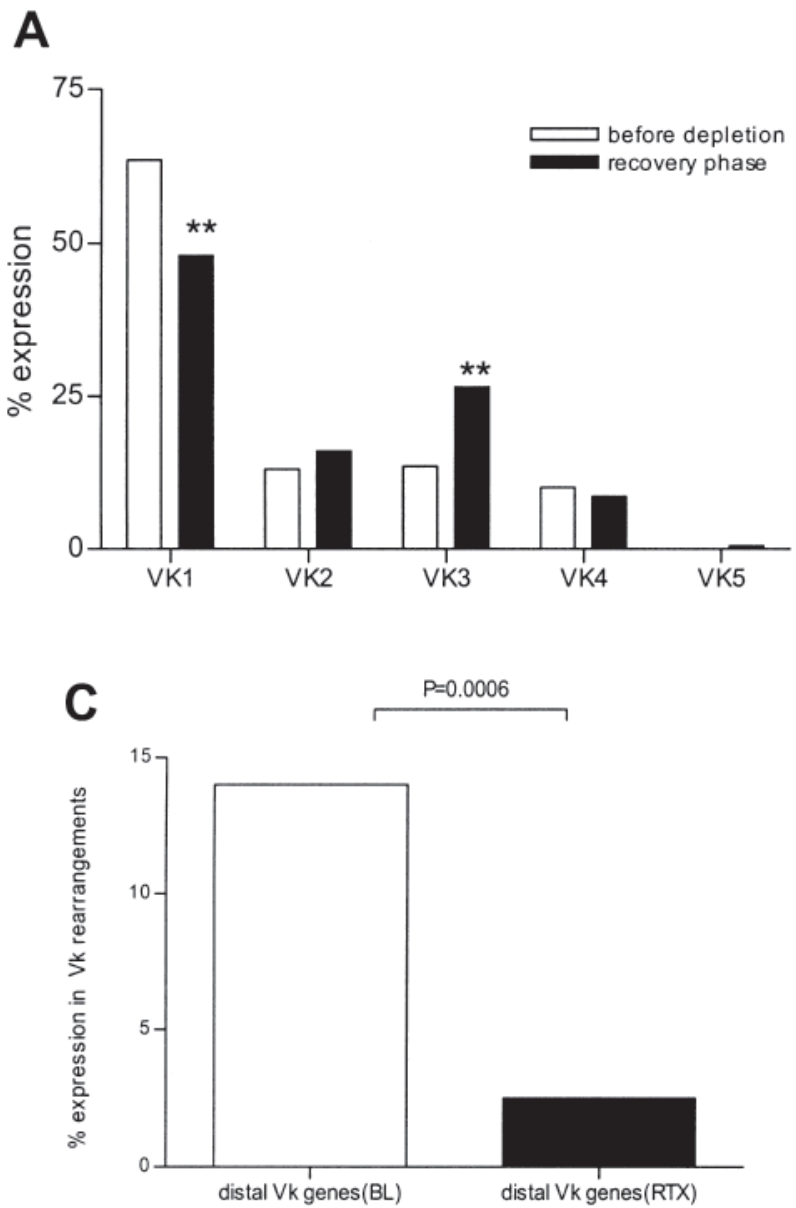

lowed by $\mathrm{V} \lambda 1$ predominantly used genes before and after therapy. V $\lambda 6,7,8$, and 9 family members were observed at very low percentages. Usage of $\mathrm{V} \lambda$ families remained mainly stable between baseline and the followup after B cell depletion (Figure 2A).

Of note, the relation between $\mathrm{V} \kappa$ and $\mathrm{V} \lambda$ gene usage was comparable between the 2 timepoints ( $52 \% \mathrm{V \kappa}$ vs $50 \%$ during regeneration; $48 \% \mathrm{~V} \lambda$ vs $50 \%$ during regeneration), excluding the possibility that RTX may have led to different usage of the 2 light chain gene repertoires.

Usage of Ig $V \kappa$ and $V \lambda$ minigenes by the CD19+/ CD27- naive $B$ cell repertoire. Analysis of individual $\mathrm{V}_{\mathrm{L}}$ minigene usage revealed that a wide range of $\mathrm{V} \kappa$ genes were employed by CD27- B cells and showed a number of changes after therapy (Figure 3). Certain genes, such as

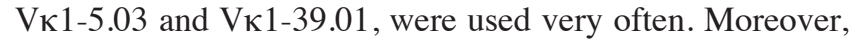
minigenes like VК1-6.01, 1D-8.01, 1D-13.01, 1-37.01, and
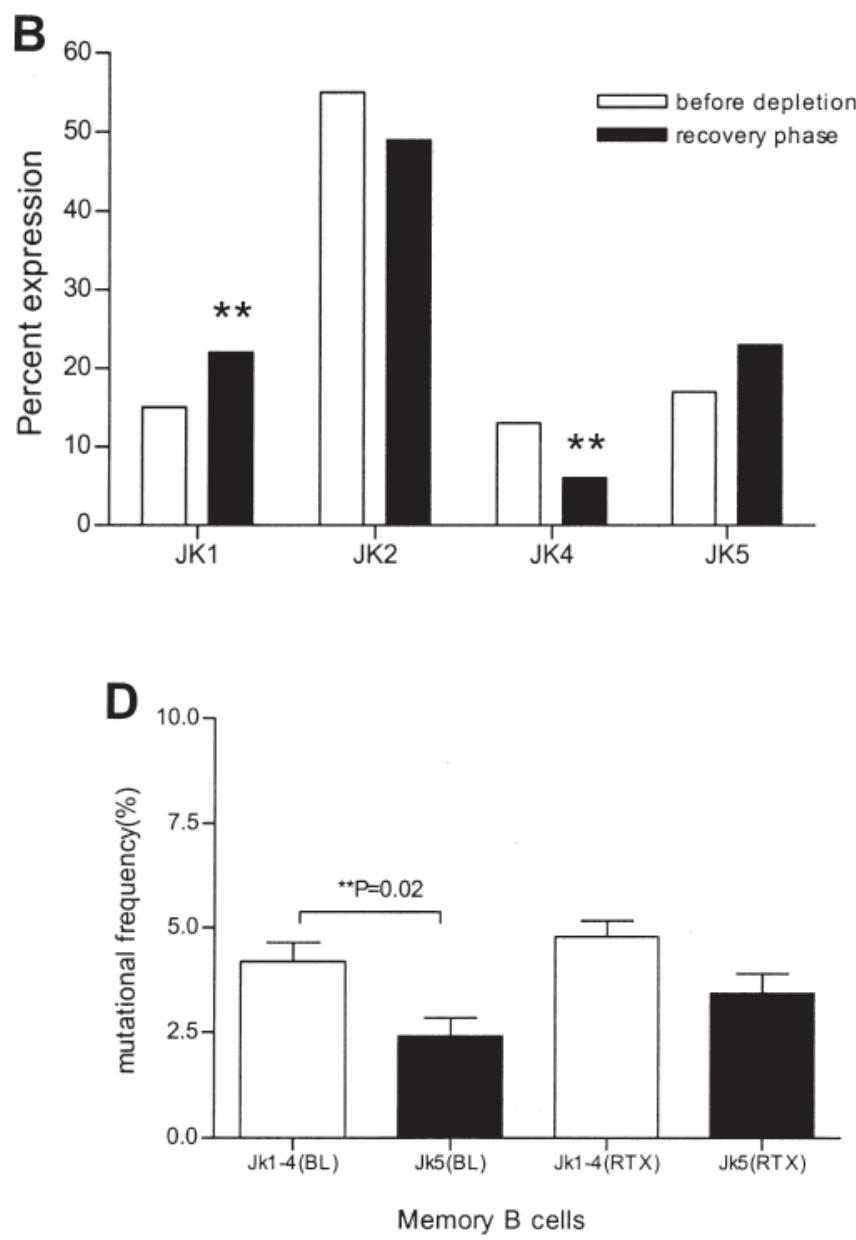

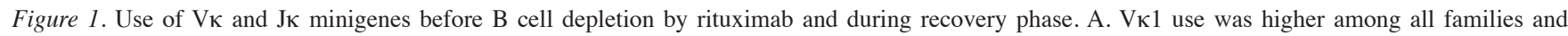

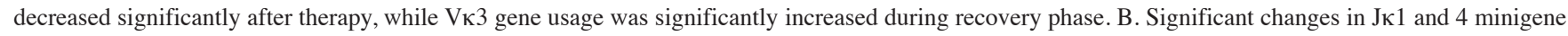

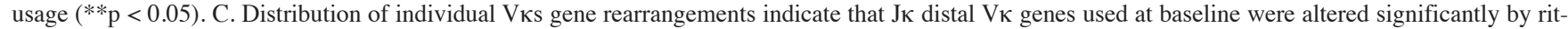

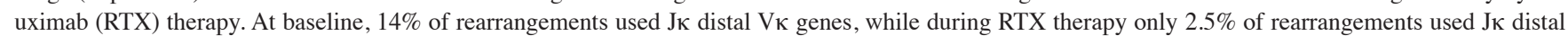

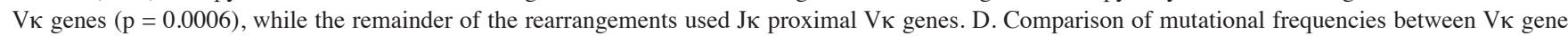

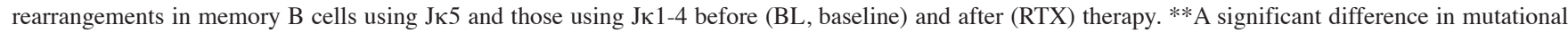

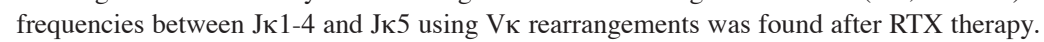

Personal non-commercial use only. The Journal of Rheumatology Copyright @ 2012 . All rights reserved. 

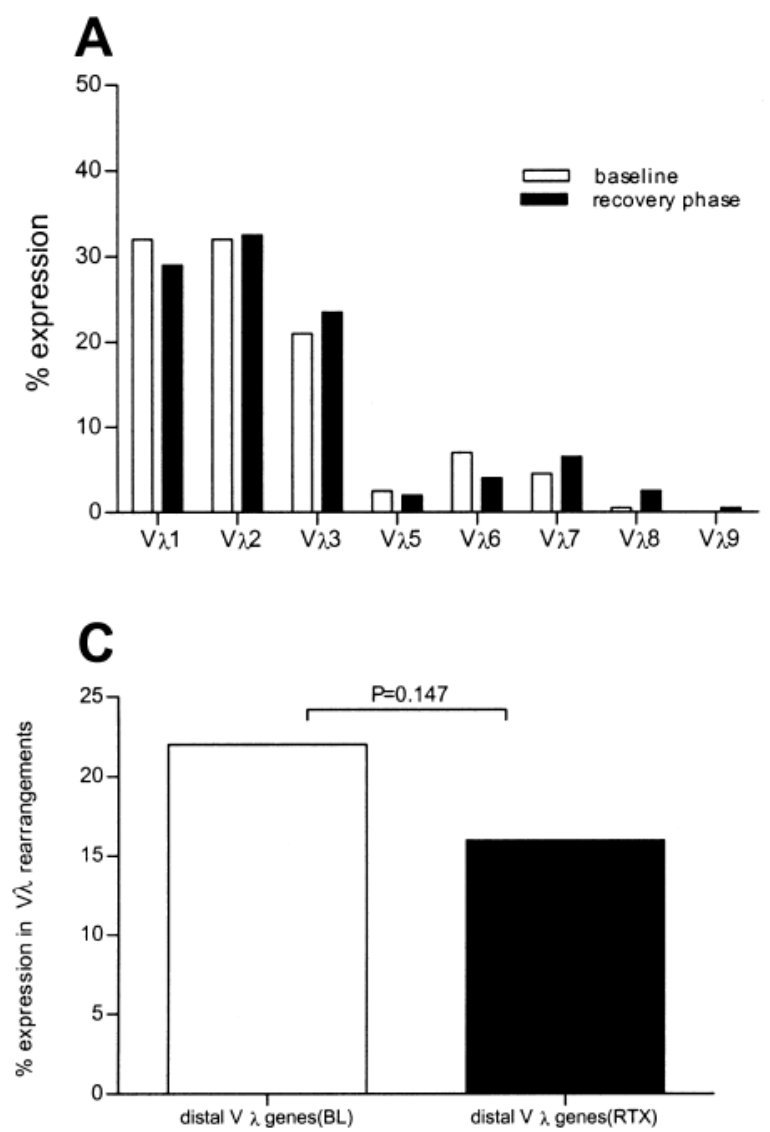

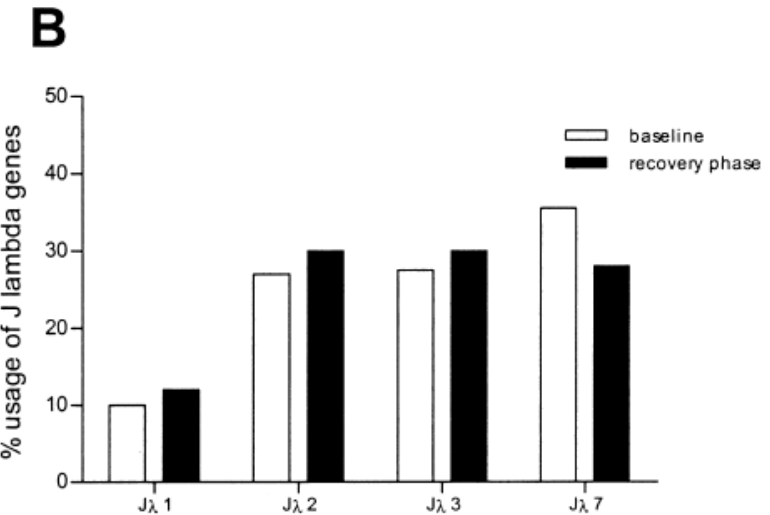

D

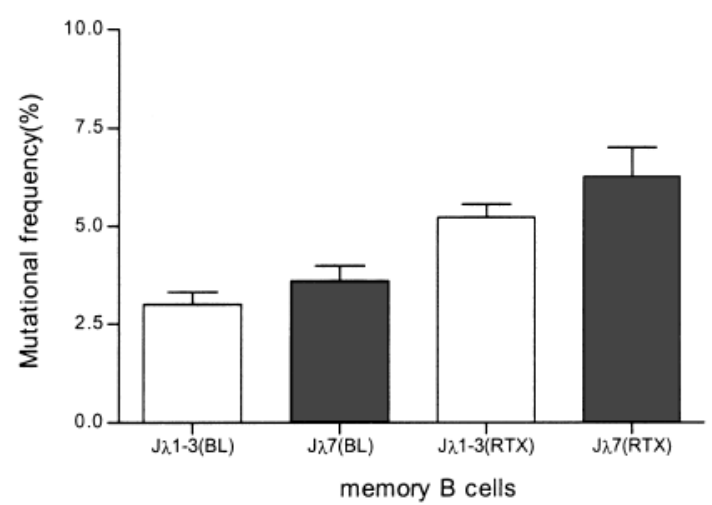

Figure 2. V $\lambda$ gene usage and $\mathrm{J} \lambda$ gene usage in RA. A. V $\lambda$ family gene usage was not substantially influenced by rituximab (RTX) therapy. B. Usage of J $\lambda$ genes after RTX was not affected by therapy. C. No significant change of usage of distal V $\lambda$ genes was found after RTX. D. Comparison of mutational frequencies between $\mathrm{V} \lambda$ gene rearrangements using J $\lambda 1-3$ and those using $\mathrm{J} \lambda 7$ before (BL, baseline) and after (RTX) therapy.

3-7.01 were expressed at lower frequencies before therapy, but were not found after therapy. By contrast, the genes Vк1-13.01, 1-13.02, 1-16.01, 2-24.01, 2-30.01, 2-40.01, and 5-2.01 were not detected before depletion, but occurred in the regenerating repertoire (Figure 3A). As expected, most of the CD27- naive B cells were unmutated, with a small number of sequences carrying very low mutations (21 sequences with 1-3 mutations, 2 sequences with 6-10 mutations out of 172 sequences).

Usage of individual $\mathrm{V} \lambda$ genes among naive $\mathrm{B}$ cells revealed a predominant expression of $\mathrm{V} \lambda 1-44.01$ and 2-14.04. All genes observed before therapy were also identified in the reemerging repertoire. In addition, several genes such as V $\lambda 1-51.01,2-11.03,3-9.01,3-21.01$, and 9-49.01 that were not found before therapy were identified after therapy. However, the frequency of occurrence of V $\lambda 3$-21.01 was significantly higher after RTX compared to baseline $(p=0.01)$. With the exception of V $\lambda 3-21.01$, there was no statistically significant difference of $\mathrm{V} \lambda$ gene usage within naive $\mathrm{B}$ cells.

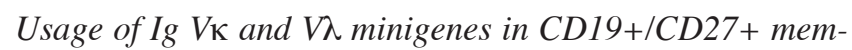

ory B cell repertoire. Analysis of the CD27+ memory B cell compartment reflecting the superimposed picture of $\mathrm{B}$ cell activation, differentiation, and selection provided further interesting insights. In terms of individual VK gene usage, Vк1-5.03, 1-39.01, and 4-1.01 genes were highly expressed before as well as after therapy. Several genes were found at lower or enhanced frequencies at the 2 timepoints of analysis, among which usage of 1-5.03 and 2-30.01 were significant after RTX (Figure 3).

Examination of individual $\mathrm{V} \lambda$ genes revealed a wide array of genes constituting the repertoire before and after depletion, i.e., V $\lambda 1-40.01,1-44.01,1-47.01,2-8.01$, 2-14.01, 2-23.01, 3-21.02, and 6-57.01. Some genes (V $\lambda 1-40.03,2-23.02,3-9.01,3-12.02,3-25.01,3-25.03$, 5-39.01, and 8-61.01) that were not found in circulation before therapy were detected in the recovery phase, albeit at lower levels, except a few significant differences (Figure 3D) of occurrence of individual $\mathrm{V} \lambda$ minigenes before and after therapy.

Imprints of potential receptor revision: usage of $\mathrm{J} \kappa$ proximal and $J_{\kappa}$ distal $V$ gene cassette. Receptor revision occurs 

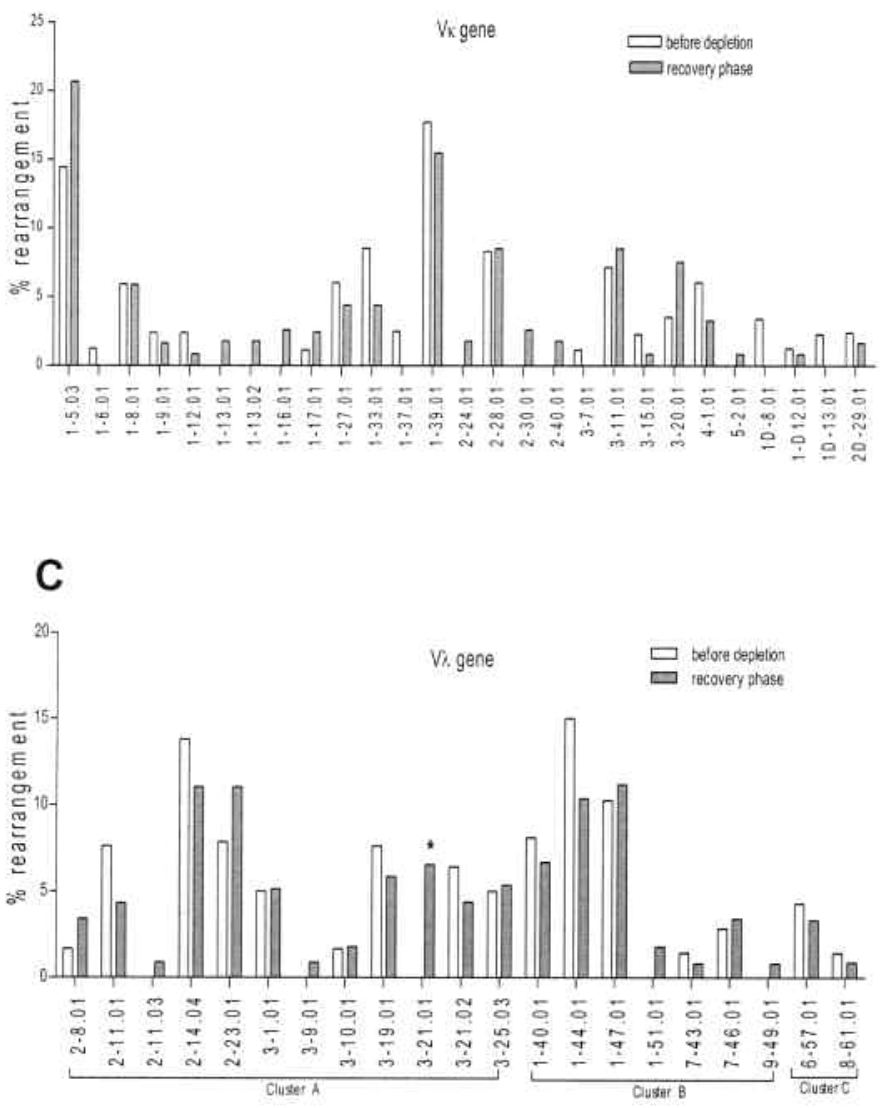

B

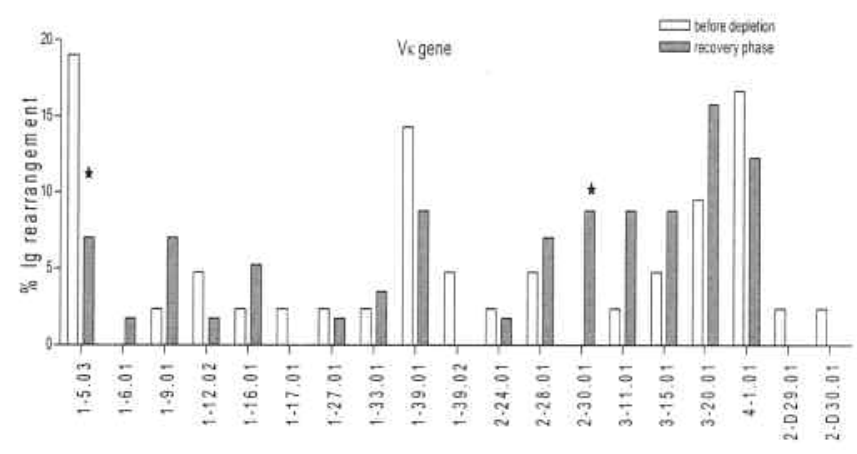

D

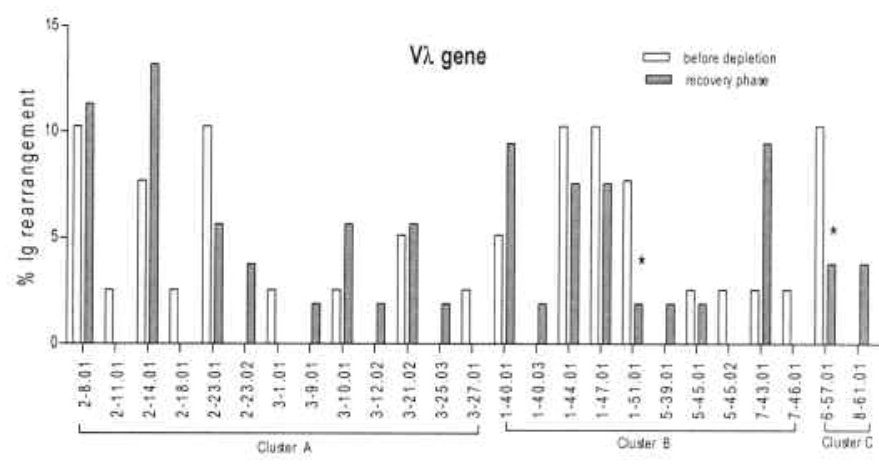

Figure 3. Light chain family minigene distribution in CD19+/CD27- (A, C) and CD19+/CD27+ (B, D) B cells before and after B cell depletion. Panels A and B represent $\mathrm{V} \kappa$ family minigene distribution before and after transient B cell depletion in naive (A) and memory B cells (B). Panels C and D display V $\lambda$ gene usage in naive and memory B cells, respectively. ${ }^{*} \mathrm{p} \leq 0.01$ compared to before therapy, 2-tailed Fisher exact test.

mainly at the Igא locus through $\mathrm{V}-$ to $\mathrm{J} \kappa$ secondary rearrangement. We further investigated whether the use of $\mathrm{V}$ genes between the proximal versus distal cassette and their rearrangements to particular $\mathrm{J}_{\kappa}$ elements differed, which may reflect active receptor revision. The JK proximal cassette is arranged when most rearrangements occur by deletion, whereas the $\mathrm{J} \kappa$ distal cassette is arranged during recombination processes by inversion and in opposite orientation to that of the proximal cassette. Among JК genes, Јк5 gene is most distally located. Analysis of $\mathrm{J} \kappa$ gene usage (Figure 1B) revealed that $\mathrm{J} \kappa 2$ was used most frequently before and after RTX. Moreover, RTX led to enhanced Jк1 usage $(15 \%$ vs $22 \% ; \mathrm{p}=0.05)$ and a reduced frequency of $\mathrm{J} \kappa 4$ gene usage ( $13 \%$ before vs $6 \%$ after RTX; $\mathrm{p}<0.025$ ). Because receptor revision leads to greater usage of $\mathrm{V} \kappa$ genes from $\mathrm{J} \kappa$ distal cassettes, the frequency of these genes was assessed. Although the overall use of JK5 gene segments (as an important indicator for active receptor revision) was not substantially different between the 2 timepoints (Figure 1B), RTX treatment led to significantly reduced usage of distal Vк genes (2.5\%) compared to baseline (14\%; $\mathrm{p}=0.0006$; Figure 1C). This indicated that the imprints of receptor revi- sion were likely more active at baseline, compared to the period after RTX. Consistent with this, the mutational frequencies between VK rearrangements of CD19+CD27+ memory B cells employing JК5 were significantly lower than those using J $1-4$ ( $\mathrm{p}=0.02$; Figure 1D).

Usage of $V \lambda$ minigenes according to their V $V \lambda$ clusters and $J \lambda$ gene usage. Further studies investigated to what extent the usage of certain $\mathrm{V} \lambda$ and $\mathrm{J} \lambda$ genes may indicate a different role of receptor revision before and after RTX. Therefore, we analyzed V $\lambda$ gene usage in both subsets according to $\mathrm{V} \lambda$ gene clusters. In this regard, $\mathrm{V} \lambda$ genes are segregated into 3 clusters, A, B, and C, cluster A being the most $\mathrm{J} \lambda$ proximal and cluster $\mathrm{C}$ being the most $\mathrm{J} \lambda$ distal segment ${ }^{20}$. Notably, the frequencies of $\mathrm{V} \lambda$ gene usage residing in cluster $\mathrm{A}, \mathrm{B}$, and $\mathrm{C}$ genes were comparable at baseline and during the reconstitution phase after RTX (Figure 3C,

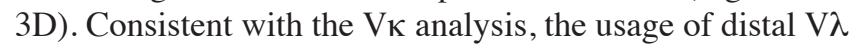
genes was reduced after RTX therapy (22\% at baseline vs $16 \%$ after RTX; $p=0.147$; Figure 2C), indicating that receptor revision was likely more active at baseline.

The frequency of usage of $\mathrm{J} \lambda$ elements was also comparable at baseline and after RTX, with moderate variations as Personal non-commercial use only. The Journal of Rheumatology Copyright @ 2012. All rights reserved. 
indicated by an increase of $\mathrm{J} \lambda 1,2$, and 3 gene usage and decreased use of $\mathrm{J} \lambda 7$ genes (not statistically significant; Figure $2 \mathrm{~B}$ ). These data indicated that $\mathrm{B}$ cell depletion by RTX led to some changes in $\mathrm{V} \lambda / \mathrm{J} \lambda$ gene usage; however, these were not significant. Moreover, analysis of the mutational frequency of $\mathrm{J} \lambda 1-3$ containing $\mathrm{V} \lambda$ rearrangements was comparable to those rearrangements employing $\mathrm{J} \lambda 7$ (Figure 2D). Together, the data indicate that there were no striking changes of receptor revision of $\mathrm{V} \lambda$ expressing $\mathrm{B}$ cells in these patients with RA undergoing RTX treatment.

Frequency of somatic hypermutation in $V \lambda$ and $V \kappa$ repertoire of $C D 27+$ memory $B$ cells. There were substantial differences in the mutational frequency of the CD19+/CD27+ memory repertoire before compared to after anti-CD20 therapy. As expected, nearly all of the CD27+ sequences were mutated, with comparable mutational frequencies of the $\mathrm{VK}$ and $\mathrm{V} \lambda$ repertoires. Notably, CD27+ memory B cells showed an almost 2-fold increase of the mutational frequency of $\mathrm{V} \lambda$ and $\mathrm{V} \kappa$ rearrangements after RTX therapy (5.6\%; Figure 4A) compared to that before therapy $(3.2 \%$; $\mathrm{p}<$
0.0001 ), with $\mathrm{V \kappa} 3.2 \% \pm 0.9 \%$ at baseline versus $5.6 \% \pm$ $0.1 \%$ in the repletion phase and $\mathrm{V} \lambda 3.1 \% \pm 0.1 \%$ versus $5.6 \% \pm 0.1 \%$. This increment of the mutational frequency was related to enhanced mutations residing in framework regions (FR 1 to 3 ) as well as complementary determining regions (CDR 1 and 2; Figure 4B, 4C). Further examination of the expression of mutations in the CDR and framework regions showed a reduced ratio of replacement to silent mutations $(\mathrm{R} / \mathrm{S})$ in the CDR $(\mathrm{p}=0.001)$ and in the FR $(\mathrm{p}=$ $0.04)$ after treatment. No differences were observed in the frequency of transitions versus transversions or in the RGYW/WRCY hotspot motif (data not shown) targeted during B cell repletion.

CDR3 length. The overall CDR3 lengths were $30.0 \pm 0.27$ and $27.0 \pm 0.16$ (median \pm SEM) within the VK rearrangements by naive and memory $\mathrm{B}$ cell rearrangements at baseline, respectively. These CDR3 lengths remained unaltered after anti-B cell therapy $(30.0 \pm 0.31$ for naive B cells and $27.0 \pm 0.26$ for memory B cells). As well, the CDR3 lengths of $\mathrm{V} \lambda$ rearrangements were similar in both naive and mem-
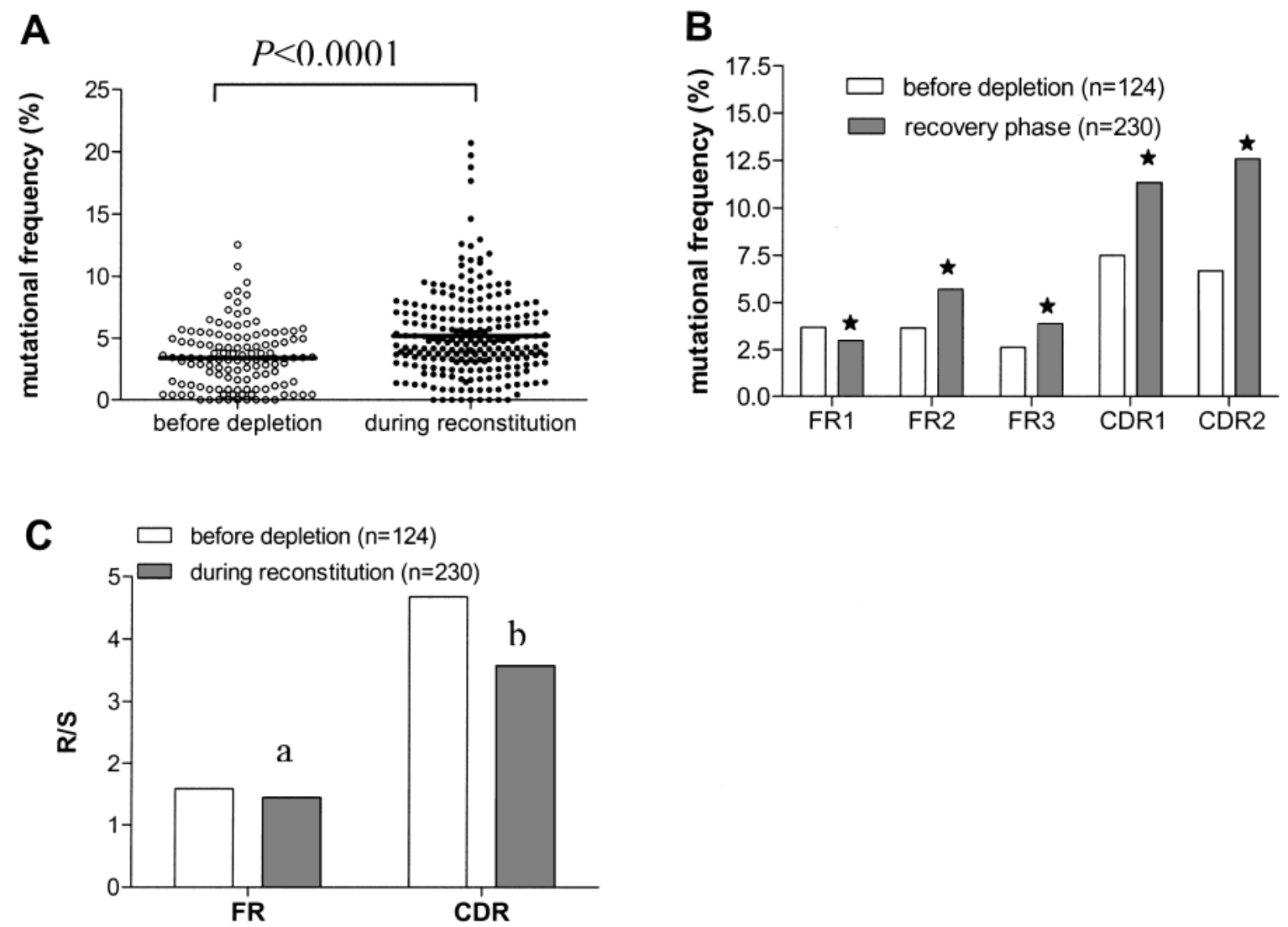

Figure 4. Pattern of mutations in the CD19+/CD27+ repertoire. A. Ig mutational frequencies of single CD19+/CD27+ B cells from the $\mathrm{V}_{\mathrm{L}}$ repertoire before $\mathrm{B}$ cell depletion and during recovery. Recovering B cells exhibit an increased mutational frequency compared to status before depletion ( $\mathrm{p}=0.0001$, chi-square test). B. Analysis of mutational frequencies in framework regions (FR) 1-3 and complementary determining regions (CDR) 1 and 2 displayed elevated mutational frequencies in CDR1 and 2 before depletion and during recovery. B cell depletion induced changes in mutational frequencies in the FR and CDR. Regenerating B cells displayed decreased FR1 mutational frequency but increased FR2, FR3, CDR1, and CDR2 frequencies. All changes were statistically significant ( $p=0.0001$, chi-square test). C. R/S mutations are diminished in the FR and CDR of CD27+ VL repertoire ( $a: p=0.04 ; b: p=0.001$, chi-square test).

Personal non-commercial use only. The Journal of Rheumatology Copyright @ 2012 . All rights reserved. 
ory B cell subsets $(33.0 \pm 0.34$ vs $30.0 \pm 0.26$ in naive; 27.0 \pm 0.40 vs $30.0 \pm 0.30$ in memory B cells) before and after RTX. The lack of changes in CDR3 lengths suggests that selective pressures effective on the CDR3 length were less strikingly different before and after RTX therapy.

\section{DISCUSSION}

B cell depletion by the anti-CD20 antibody RTX has proven to be an efficacious and comparably safe treatment in patients with moderate to severe RA as well as other autoimmune diseases ${ }^{7,8,21}$, supporting the role of B cells in their pathophysiology. RTX-mediated B cell depletion yields a longterm effect on immunological homeostasis including distinct peripheral blood B cell subpopulations with high numbers of transitional B cells ${ }^{8}$, indicating recapitulation of $\mathrm{B}$ cell ontogeny. Although numeric normalization of B cell numbers takes place in the majority of patients between 1 and 2 years after a single course of RTX, a longterm delay in memory B cell subsets has been observed especially in responders ${ }^{8,9}$. Gene rearrangement analysis of $\operatorname{IgV}$ using single B cells allows study of different processes such as somatic hypermutation and receptor revision. Abnormalities in these events alter the composition of the peripheral B cell repertoire. Previous observations provided evidence of the influence of RTX on molecular imprints shaping the Ig heavy chain receptor ${ }^{15,22,23}$, but did not allow any evaluation of receptor revision. Receptor revision together with the imprints of somatic hypermutation, CDR3 length, and Ig switching represent results of $\mathrm{T}$ cell and antigen-dependent $\mathrm{B}$ cell activation and differentiation, which also reflect molecular signatures of immune memory. To date, modulatory effects in the immunoglobulin light chain repertoire after RTX have not been studied in detail. Therefore in this

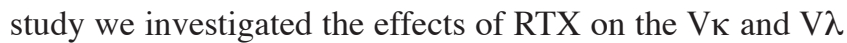
light chain repertoire genes in the CD27- and CD27+ B cell compartment in patients with active RA, using single $\mathrm{B}$ cell analyses before and during B cell repopulation after RTX.

Different mechanisms shaping the human light chain repertoire including selection and combinational biases serve the limitation of expressed repertoire. Repertoire revision in B cells and the clonal selection processes contribute to somatic evolution of appropriate immune responses under normal circumstances ${ }^{6}$. Examination of individual Vк minigene distribution revealed a diverse repertoire of genes, with specific VК1 family members being predominantly expanded in repopulating B cells. A similar overrepresentation of Vк1 family members has also been reported in cDNA libraries studied from synovial tissue of a patient with RA in comparison to healthy donors ${ }^{24}$. A restricted repertoire of Vк has been reported in patients with juvenile arthritis ${ }^{11}$. Interestingly, we did not find a restricted repertoire of $\mathrm{VK}$ family gene usage in our patients with RA, which may indicate an age-dependent modulation (Figure 1A, 1B). The Vא family repertoire changed significantly after RTX (Figure
$3 \mathrm{~A}$ ) as indicated by a decrease in the use of $\mathrm{V} \kappa 1$ and an increase in Vк3 families. Interesting as well, the B3 gene, a member of the VК4 family, previously shown to be overexpressed in patients with systemic lupus erythematosus $(\mathrm{SLE})^{25}$ and linked to encode autoantibodies ${ }^{26}$, was not used by our 2 patients with RA, consistent with a differential usage of $\mathrm{VK}$ minigenes during flares of different autoimmune diseases. Analysis of the $\mathrm{V} \lambda$ repertoire showed the majority of minigenes in equal distributions after RTX, except V $\lambda 3-21.01$, which was used significantly more $(\mathrm{p}=$ 0.01) in repopulated CD27- B cells, while 1-51.01 and 6-57.01 were reduced in CD27+ B cells after RTX. These findings indicate that a diverse and polyclonal $\mathrm{V} \kappa$ and $\mathrm{V} \lambda$ gene repertoire is emerging after RTX-induced temporal B cell depletion, suggesting a reestablishment of $\mathrm{B}$ cell homeostasis.

There is controversy whether defective mechanisms in secondary gene rearrangements are involved in generating autoimmunity. Studies indicate that receptor revision is a principal mechanism of $\mathrm{B}$ cell tolerance ${ }^{12}$ and defective central or peripheral receptor revision has been linked to autoimmunity ${ }^{27}$. The frequency of J-distal $\mathrm{V} \kappa$ gene usage was significantly less than J-proximal VK genes after therapy (Figure 1C). These data indicate that imprints of receptor revision are already evident before RTX, and therapy-related changes do not support a potential defective receptor revision in patients with RA. Two different molecular mechanisms are operative on $\mathrm{V} \kappa / \mathrm{J} \kappa$ rearrangements, one by inversion and another by deletion. Inversion is believed to be the significantly less efficient process, suggested by artificial recombination substrate studies ${ }^{28,29}$. Recombination requires rearrangement over a long stretch of DNA because the J-distal cassette is 1500 to $2000 \mathrm{~kb}$ from the nearest $\mathrm{JK}$ gene, with possibly less efficient recombination machinery over distances $>1000 \mathrm{~kb}$. While no firm conclusion can be drawn about the exact mechanisms applied by patients with RA, the current data for significantly reduced usage of JK-distal genes in repopulated B cells suggest that RTX therapy normalizes B cell homeostasis by reducing the imprints of receptor revision, which may have been active particularly on $\mathrm{V} \kappa \mathrm{J} \kappa$ gene rearrangements. Because of the distal location of $\mathrm{J} \kappa 5$, a diminished mutational frequency was observed in memory B cell rearrangements compared to those using Jא1-4 (Figure 1D) at baseline. Together, the data indicate that receptor revision took place at baseline on the Vк locus, excluding the possibility of a defective receptor revision process in these patients with RA. Moreover, receptor revision in Vк probably occurred in secondary lymphoid organs after initiation of somatic hypermutation, as suggested by fewer mutations in rearrangements using Јк5.

Analysis of the $\mathrm{V} \lambda$ gene repertoire did not reveal significant differences after RTX. The overall usage of J-distal V $\lambda$ genes was decreased without reaching statistical significance (Figure 4C), indicating that the effects of RTX on V $\lambda$ 
gene rearrangements were less striking compared to the Vк locus. This is consistent with the conclusion that receptor revision in $\mathrm{V} \lambda$ occurred centrally at baseline.

We did not find increased usage of cluster $\mathrm{C}$ genes, in contrast to previous findings in a patient with $\mathrm{SLE}^{30}$. Increased use of cluster $\mathrm{C}$ genes is believed to be a predictor of extensive receptor revision in $\mathrm{V} \lambda$ gene rearrangements because it is also observed in infants compared to healthy subjects ${ }^{31}$. Cluster $\mathrm{C}$ genes are most $\mathrm{J} \lambda$ distally located with more than $600 \mathrm{~kb}$ upstream of $\mathrm{J} \lambda 1$ locus. In our patients with $\mathrm{RA}$, cluster A and B genes were frequently used, consistent with findings from healthy donors ${ }^{31}$. Only moderate changes were observed in the usage of $J \lambda$ genes during therapy (Figure 2B), suggesting that the basic molecular mechanisms driving recombination and selection of $\mathrm{V} \lambda$ chain rearrangements were not substantially altered by RTX.

We noted profound changes in the $\mathrm{CD} 27+$ memory $\mathrm{B}$ cells during B cell repopulation after RTX. The mutational pattern of repopulated CD27+ memory B cells was marked by the prevalence of highly mutated $\mathrm{V} \kappa$ and $\mathrm{V} \lambda$ Ig gene sequences (Figure 4A). These findings are in accord with our previous findings, where we analyzed the mutational pattern of $\mathrm{Ig}-\mathrm{V}_{\mathrm{H}} 4$ gene in a cohort of RA patients treated with $\mathrm{RTX}^{15}$, and support the concept that RTX-mediated B cell depletion modulates Ig heavy and light chains. It is assumed that repopulation of the peripheral repertoire is mainly derived from early undepleted CD20-negative pro-B cells, and permits early occurrence of highly mutated sequences during B cell repopulation. These highly mutated cells during the early regeneration phase might develop from a newly generated B cell pool that rapidly undergoes intensive somatic hypermutation and circulates in the periphery. However, part of the highly mutated memory pool may also be dependent on surviving cells, which can be found in the periphery in low frequency during the depletion phase and may generate from protected niches ${ }^{32,33}$. The increased mutational frequency by regenerated memory $\mathrm{B}$ cells can be explained by a marked reduction in pre-switch memory B cells and a relative increase of recirculating plasmablasts during the regeneration phase (Table 1). Detailed analyses of CDR (Figure 4B) showed that the V $\lambda$ and $\mathrm{VK}$ repertoire exhibited increased mutational frequency in the CDR2, followed by CDR1, before and after depletion. Similar observations were noted within framework regions. This indicates that B cell depletion by RTX introduced significant modulations in the regenerating $\mathrm{V} \lambda / \mathrm{V} \kappa$ repertoire. Of note, the ratio of replacement to silent mutations $(\mathrm{R} / \mathrm{S})$ in the complementary determining regions and framework regions revealed that RTX induced modulations of R/S ratios. We observed a significantly decreased $\mathrm{R} / \mathrm{S}$ mutational ratio after RTX (R/S CDR and FR in $\mathrm{V}_{\mathrm{L}}: \mathrm{p}=0.001, \mathrm{p}=$ 0.04 , baseline vs reconstitution phase, respectively). Positive selection of $\mathrm{R}$ mutations has been reported for $\mathrm{V}_{\mathrm{H}}$ and $V_{L}$ gene rearrangements in healthy individuals $34,35,36$, and therefore the reduction in R/S mutations may also be indicative of lower affinity of potential autoreactive clones that recirculate during $\mathrm{B}$ cell reconstitution in patients with RA.

Overall, this is to our knowledge the first study to report the regeneration pattern of $\mathrm{Ig} V \kappa$ and $\mathrm{V} \lambda$ light chain gene rearrangements during RTX therapy. Receptor revision was operative in these patients mainly on the VK locus at baseline. Transient B cell depletion introduced differential VкJK and $V \lambda J \lambda$ minigene usage in the regenerating naive and memory B cell repertoires. Memory B cells were substantially marked by an elevated mutational frequency of the reemerging CD27+ light chain. While our findings do not support defects of receptor revision, at least on the VK locus in these patients with RA, the data provide evidence that anti-CD20 therapy probably alters the molecular characteristics of the $\mathrm{V}$ light chain gene repertoire consistent with a reduction of receptor revision.

\section{ACKNOWLEDGMENT}

We thank Katharina Eckert, Anette Koss-Kinzinger, Isabelle Kuntzsch, and Christian Linden for their excellent technical assistance.

\section{REFERENCES}

1. Firestein GS. Evolving concepts of rheumatoid arthritis. Nature 2003;423:356-61.

2. Marston B, Palanichamy A, Anolik JH. B cells in the pathogenesis and treatment of rheumatoid arthritis. Curr Opin Rheumatol 2010;22:307-15.

3. Takemura S, Klimiuk PA, Braun A, Goronzy JJ, Weyand CM. T cell activation in rheumatoid synovium is B cell dependent. J Immunol 2001;167:4710-8.

4. Dorner T. Crossroads of B cell activation in autoimmunity: Rationale of targeting B cells. J Rheumatol Suppl. 2006 May;77:3-11.

5. Hansen A, Lipsky PE, Dorner T. B-cell lymphoproliferation in chronic inflammatory rheumatic diseases. Nat Clin Pract Rheumatol 2007;3:561-9.

6. Dorner T, Lipsky PE. Molecular basis of immunoglobulin variable region gene usage in systemic autoimmunity. Clin Exp Med 2005;4:159-69.

7. Edwards JC, Szczepanski L, Szechinski J, Filipowicz-Sosnowska A, Emery P, Close DR, et al. Efficacy of B-cell-targeted therapy with rituximab in patients with rheumatoid arthritis. N Engl J Med 2004;350:2572-81.

8. Roll P, Palanichamy A, Kneitz C, Dorner T, Tony HP. Regeneration of B cell subsets after transient B cell depletion using anti-CD20 antibodies in rheumatoid arthritis. Arthritis Rheum 2006; 54:2377-86.

9. Anolik JH, Friedberg JW, Zheng B, Barnard J, Owen T, Cushing E, et al. B cell reconstitution after rituximab treatment of lymphoma recapitulates B cell ontogeny. Clin Immunol 2007;122:139-45.

10. Wardemann H, Yurasov S, Schaefer A, Young JW, Meffre E, Nussenzweig MC. Predominant autoantibody production by early human B cell precursors. Science 2003;301:1374-7.

11. Morbach H, Richl P, Faber C, Singh SK, Girschick HJ. The kappa immunoglobulin light chain repertoire of peripheral blood B cells in patients with juvenile rheumatoid arthritis. Mol Immunol 2008;45:3840-6.

12. Halverson R, Torres RM, Pelanda R. Receptor editing is the main mechanism of $\mathrm{B}$ cell tolerance toward membrane antigens. Nature

$$
\text { Personal non-commercial use only. The Journal of Rheumatology Copyright (c) 2012. All rights reserved. }
$$


Immunol 2004;5:645-50.

13. Samuels J, Ng YS, Coupillaud C, Paget D, Meffre E. Impaired early B cell tolerance in patients with rheumatoid arthritis. J Exp Med 2005;201:1659-67.

14. Arnett FC, Edworthy SM, Bloch DA, McShane DJ, Fries JF, Cooper NS, et al. The American Rheumatism Association 1987 revised criteria for the classification of rheumatoid arthritis. Arthritis Rheum 1988;31:315-24.

15. Palanichamy A, Roll P, Theiss R, Dorner T, Tony HP. Modulation of molecular imprints in the antigen-experienced B cell repertoire by rituximab. Arthritis Rheum 2008;58:3665-74.

16. Frolich D, Giesecke C, Mei HE, Reiter K, Daridon C, Lipsky PE, et al. Secondary immunization generates clonally related antigen-specific plasma cells and memory B cells. J Immunol 2010;185:3103-10.

17. Brezinschek HP, Foster SJ, Dorner T, Brezinschek RI, Lipsky PE. Pairing of variable heavy and variable kappa chains in individual naive and memory B cells. J Immunol 1998;160:4762-7.

18. Farner NL, Dorner T, Lipsky PE. Molecular mechanisms and selection influence the generation of the human V lambda $\mathrm{J}$ lambda repertoire. J Immunol 1999;162:2137-45.

19. Souto-Carneiro MM, Longo NS, Russ DE, Sun HW, Lipsky PE. Characterization of the human Ig heavy chain antigen binding complementarity determining region 3 using a newly developed software algorithm, JOINSOLVER. J Immunol 2004;172:6790-802.

20. Frippiat JP, Williams SC, Tomlinson IM, Cook GP, Cherif D, Le Paslier D, et al. Organization of the human immunoglobulin lambda light-chain locus on chromosome 22q11.2. Human Mol Genet 1995;4:983-91.

21. Lindholm C, Borjesson-Asp K, Zendjanchi K, Sundqvist AC, Tarkowski A, Bokarewa M. Longterm clinical and immunological effects of anti-CD20 treatment in patients with refractory systemic lupus erythematosus. J Rheumatol 2008;35:826-33.

22. Muhammad K, Roll P, Einsele H, Dorner T, Tony HP. Delayed acquisition of somatic hypermutations in repopulated IGD+CD27+ memory B cell receptors after rituximab treatment. Arthritis Rheum 2009;60:2284-93.

23. Rouziere AS, Kneitz C, Palanichamy A, Dorner T, Tony HP. Regeneration of the immunoglobulin heavy-chain repertoire after transient B-cell depletion with an anti-CD20 antibody. Arthritis Res Ther 2005;7:R714-24.
24. Pyon HS, Ha-Lee YM, Song GG, Sohn J. Analysis of Ig kappa light chain gene variable regions expressed in the rheumatoid synovial B cells. Scand J Immunol 2001;53:503-9.

25. Dorner T, Foster SJ, Farner NL, Lipsky PE. Immunoglobulin kappa chain receptor editing in systemic lupus erythematosus. J Clin Invest 1998;102:688-94.

26. Meffre E, Davis E, Schiff C, Cunningham-Rundles C, Ivashkiv LB, Staudt LM, et al. Circulating human B cells that express surrogate light chains and edited receptors. Nat Immunol 2000;1:207-13.

27. Prak EL, Weigert M. Light chain replacement: A new model for antibody gene rearrangement. J Exp Med 1995;182:541-8.

28. Foster SJ, Brezinschek HP, Brezinschek RI, Lipsky PE. Molecular mechanisms and selective influences that shape the kappa gene repertoire of IgM+ B cells. J Clin Invest 1997;99:1614-27.

29. Gauss GH, Lieber MR. The basis for the mechanistic bias for deletional over inversional V(D)J recombination. Genes Dev 1992;6:1553-61.

30. Dorner T, Farner NL, Lipsky PE. Ig lambda and heavy chain gene usage in early untreated systemic lupus erythematosus suggests intensive B cell stimulation. J Immunol 1999;163:1027-36.

31. Lee J, Monson NL, Lipsky PE. The V lambda J lambda repertoire in human fetal spleen: Evidence for positive selection and extensive receptor editing. J Immunol 2000;165:6322-33.

32. Mei HE, Frolich D, Giesecke C, Loddenkemper C, Reiter K, Schmidt S, et al. Steady-state generation of mucosal IgA+ plasmablasts is not abrogated by B-cell depletion therapy with rituximab. Blood 2010;116:5181-90.

33. Hoyer BF, Moser K, Hauser AE, Peddinghaus A, Voigt C, Eilat D, et al. Short-lived plasmablasts and long-lived plasma cells contribute to chronic humoral autoimmunity in NZB/W mice. J Exp Med 2004;199:1577-84.

34. Dorner T, Foster SJ, Brezinschek HP, Lipsky PE. Analysis of the targeting of the hypermutational machinery and the impact of subsequent selection on the distribution of nucleotide changes in human VHDJH rearrangements. Immunol Rev 1998;162:161-71.

35. Foster SJ, Dorner T, Lipsky PE. Somatic hypermutation of V kappa J kappa rearrangements: Targeting of RGYW motifs on both DNA strands and preferential selection of mutated codons within RGYW motifs. Eur J Immunol 1999;29:4011-21.

36. Monson NL, Dorner T, Lipsky PE. Targeting and selection of mutations in human V lambda rearrangements. Eur J Immunol 2000;30:1597-605. 